\title{
Diurnal and stress-reactive dehydroepiandrosterone levels and telomere length in youth
}

\section{Andrew R Dismukes ${ }^{1,2}$, Vanessa J Meyer ${ }^{1,2}$, Elizabeth A Shirtcliff1, Katherine P ThealI ${ }^{2}$, Kyle C Esteves ${ }^{2}$ and Stacy S Drury ${ }^{2}$}

1'Department of Human Development and Family Studies, lowa State University, Ames, lowa, USA ${ }^{2}$ Tulane University School of Medicine, Department of Psychiatry, Division of Child and Adolescent Psychiatry, Tulane University, New Orleans, Louisiana, USA
Correspondence should be addressed to S S Drury

Email sdrury@tulane.edu

\begin{abstract}
The current investigation examined the association between the aging-related biomarkers dehydroepiandrosterone (DHEA) and telomere length (TL) in communityrecruited African-American youth. The examination of DHEA included stress reactive, basal and diurnal sampling, in order to elucidate the underlying physiological process that may overlap with TL. One hundred and two participants completed the Trier Social Stressor Test for children (TSST-C). TL was obtained from all youth from buccal swabs on the same day as the TSST-C. Saliva samples from 83 participants were obtained over the course of two additional days to measure waking and diurnal levels of DHEA. DHEA diurnal slope was a robust predictor of TL $(B=0.516, P<0.05)$, while other DHEA values were not significantly associated with TL. This study is one of the first studies to examine basal, diurnal and reactivity measurements of DHEA in youth. Furthermore, this is the first study, to our knowledge, to demonstrate a positive association between DHEA, a putative anti-aging hormone, and $\mathrm{TL}$, an indicator of cellular aging.
\end{abstract} Key Words

\section{Introduction}

Dehydroepiandrosterone (DHEA) is a steroid hormone that is sometimes called the 'anti-aging hormone' because of ts protective effects on memory, neuronal growth, and neuronal activity (1). DHEA is produced both centrally, as a neurosteroid, and peripherally by the adrenal cortex and gonads. Like other steroid hormones, DHEA levels are expected to decline across the day, with a flatter diurnal slope corresponding to an overall greater cumulative daily DHEA exposure. DHEA also fluctuates in response to social stressors (2). Unlike other steroid hormones that have specific receptors, DHEA binds to a range of receptors, including the estrogen receptor (3). DHEA serves as a prohormone for estrogen and testosterone and can broadly influence physiology through its conversion to other sex steroids and metabolites indicating the potential for a diverse pattern of downstream effects.

Similar to DHEA, telomere length (TL) has also been associated with a range of factors linked to biological aging. Telomeres are the protective caps at the ends of chromosomes that shorten with every cell division and serve as a molecular clock, with shorter TL reflecting older cellular age. TL is critical for cellular senescence and apoptosis and may serve a global regulatory function in relation to genomic stability and gene expression (4).

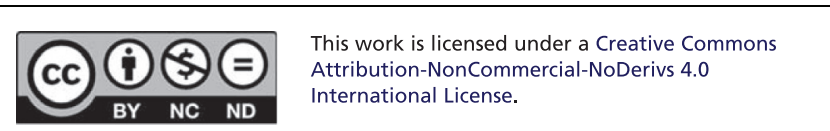




\section{DHEA: the anti-aging hormone}

DHEA is sometimes called the 'anti-aging hormone'; however, DHEA exerts effects across the life course. At one end of the aging spectrum, DHEA is positively associated with pubertal development. A steady increase in DHEA production is observed with adrenarche, the maturation of the adrenal gland (1) and the hallmark of pubertal initiation. The adrenal glands continue to produce DHEA into young adulthood, followed by a gradual decline of DHEA in the later decades of life (5). DHEA also functions at the other end of the aging process. For example, in rodents, replacement of DHEA reverses age-related dendritic changes in the CNS (6). Overall, in older humans, low DHEA levels have been associated with increased mortality in men, though in women this relationship is less clearly characterized (7). DHEA supplementation has been preliminarily linked to improvements in bone turnover and increased libido $(8,9)$ and may also be protective against coronary heart disease in men (10). These findings indicate that DHEA is mechanistically linked, at both ends of life, to biological aging and age-related conditions.

\section{TL: the molecular clock}

Like DHEA, TL exhibits different trajectories as a function of aging. Specifically, in the few longitudinal studies to date, rapid TL shortening appears to occur during the first years of life, followed by relative stabilization until the fourth or fifth decades when TL begins to demonstrate a steady age-related decline that parallels that of DHEA $(11,12)$. In addition to cellular studies where decreased $\mathrm{TL}$ is directly linked to cellular senescence, associations between shorter TL and a range of adverse age-related health outcomes have been demonstrated. Specifically, shorter TL has been linked to cardiovascular disease, diabetes, and obesity in both adults and youth $(13,14,15)$. Furthermore, in animal models, TL attrition is likely linked to longevity; longer telomeres measured early in life have been linked to longer lifespans (16).

\section{Adolescence, TL, and DHEA}

Although childhood is not typically considered a developmental time period in which aging factors are considered problematic, it is still worthwhile to explore the association between DHEA and TL during adolescence for several reasons. First, adolescence is a time period of marked reorganization of physiologic systems as well as considerable increases in DHEA levels. Second, during this time period, DHEA is coming 'on-line' to influence many of the agerelated changes of adolescence, such as the development of axillary and pubic hair, changes in sweat gland production resulting in body odor, and changes in skin oil production linked to acne. By extension, and due to the rapidly changing physiologic systems during this time period, concurrent with significant elevations of DHEA, adolescence may be a developmental time period during which TL may also be sensitive to the age-related effects of DHEA. In summary, adolescence is a critical period for neuroendocrine regulation of growth and development, making this an ideal window into the association between DHEA and TL.

\section{The present investigation: DHEA and TL}

To best characterize the relationship between TL and DHEA levels, the present investigation examined the association between TL and acute reactivity, diurnal variation and waking levels of DHEA. This characterization of both diurnal and reactivity levels of DHEA permits exploration of the differential association between TL and multiple components of the shape of the DHEA curve on both basal and reactive days, a virtue not provided by other approaches. As such, we hypothesized that increased DHEA would be associated with longer TL, but did not make a priori predictions about how DHEA reactivity or rhythm that would influence TL.

\section{Materials and methods}

\section{Participants}

One hundred and two African-American participants were included in the final analysis. A total of 120 predominantly African-American children aged 5-15 years were recruited from the greater New Orleans area (for details see (17)). Analyses excluded 11 individuals of other races. Of the remaining 109 participants, 102 participants had DHEA reactivity, recovery, and peak hormone data across the Trier Social Stress Task. DHEA waking and diurnal slope data were collected on a subset of these, resulting in 83 participants with both reactivity and diurnal variables. This study was approved by the Tulane Institutional Review Board.

\section{Measures}

Caregivers provided information during an interview with trained research assistants about multiple levels of the

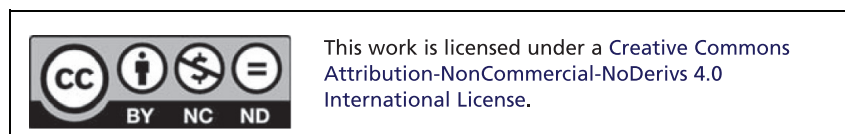


child's life and social ecology. Relevant control variables collected included body mass index, sex, age, puberty status, and maternal education.

\section{Trier Social Stressor Test for children}

The Trier Social Stressor Test for children (TSST-C) is a validated psychosocial stressor for youth (18). After a 5-min preparation period, the child was led into a separate room and told to stand in front of two unfamiliar adults and a video camera. The child was given the introduction to a story and asked to finish the story in a free speech for $5 \mathrm{~min}$. Next, the committee asked the children to serially subtract numbers out loud. On every failure, the committee interfered, stating 'That is incorrect. Please start from the beginning'.

\section{Saliva collection}

In order to capture both DHEA reactivity to a psychosocial stressor and diurnal DHEA variation, saliva was collected by passive drool during the laboratory visit and at home. On the laboratory day, the TSST-C was administered in the early afternoon (mean 14:03 h). Saliva was collected a mean of $39 \mathrm{~min}$ after arrival to the research site and then twice after the TSST-C (mean $23 \mathrm{~min}$ and mean $85 \mathrm{~min}$ post-TSST-C). Eighty-three participants provided diurnal (home) saliva samples: upon awakening (mean 08:01 h), 30 min later (mean 08:35 h), in the early afternoon (mean 15:57 h), and at bedtime (mean 20:17 h).

\section{DHEA assay}

Samples were collected by passive drool, frozen immediately, and delivered to the laboratory of Dr Elizabeth A Shirtcliff. On the day of testing, saliva was thawed and centrifuged at $1500 \boldsymbol{g}$ for $15 \mathrm{~min}$. The clear top phase was pipetted into appropriate test wells. Saliva was assayed for DHEA using a commercially available enzyme immunoassay specifically designed for use with saliva (Salimetrics, State College, PA, USA). The test uses $25 \mu \mathrm{L}$ of saliva (per singlet determination) and has a minimum detection limit of $5 \mathrm{pg} / \mathrm{mL}$. Average kit intraassay and inter-assay coefficient of variations (CVs) are 5.6 and 8.2, respectively. All samples were assayed in duplicate, and duplicate test values that varied by more than 7\% (intra-assay CV) were repeated. Final values for DHEA concentration were log transformed to normalize the distribution.

\section{Telomere length}

Buccal swabs were collected during anthropometric data collection before the TSST, immediately stored at $4^{\circ} \mathrm{C}$, and extracted within 5 days of collection. Quality control measures were done using Nanodrop and Qubit for concentration and gel electrophoresis for DNA integrity. Details on TL quantification have previously been published (17). Briefly, DNA was extracted from buccal swabs. The average relative buccal cell TL was determined from the telomere repeat copy number to single gene (albumin) copy number (T/S) ratio using quantitative real-time PCR. Measures were performed in triplicate, and triplicate plates were repeated. The average of the six replicates across both plates was used to calculate TL.

\section{Analytic approach}

Correlations were initially run between DHEA and TL. Next, a series of regression analyses were performed with DHEA and control items predicting TL as the outcome of interest. Control variables and TL were available for all participants. Control variables were selected based on known association and the potential to influence TL or DHEA levels. Regression analyses controlled for BMI, maternal education as a marker of socioeconomic status coded as 0-8 ranging in achievement from grade school to graduate school, puberty status categorized by maternal-reported Pubertal Development Scale (19) converted to tanner stage (20), child sex, and age. Sample characteristics are listed in Table 1. Puberty and age controls were not run in the same model because this would change the meaning of the control term for puberty from a developmental process to that of a main effect above and beyond age (i.e. pubertal timing), which is inconsistent with the analytic emphasis on the process of aging. The TL outcome term was adjusted for maternal and paternal age at conception (21).

DHEA variables on each individual were managed using HLM software ( $\mathrm{v}$ 6.0; Scientific Software International Inc, Skokie, IL, USA). Five unique variables were calculated for DHEA: TSST-C-peak, TSST-C-reactivity, TSST-C-recovery, awakening basal level, and diurnal slope. DHEA variables were captured as predicted scores based on Empirical Bayes 'shrunken' estimates of betweenindividual slopes-as-outcomes (level 2) derived from within-individual equations (level 1). For diurnal DHEA, each individual received a score for awakening basal DHEA level and DHEA slope across the day, respectively, by entering time (centered on waking-values) as a unique This work is licensed under a Creative Commons
Attribution-NonCommercial-NoDerivs 4.0 International License. 
predictor of each DHEA level across the 2 days. Similarly, reactivity and recovery slopes were calculated from DHEA scores measured during the TSST-C on the time-topeak and time-since-peak, respectively; this HLM model was also used to extract the peak DHEA level achieved during the stressor. Correlations and stepwise regression analyses were performed across each of the five unique terms of DHEA.

Table 1 Demographics of the sample. Values are reported as percentages or as mean (S.D.)

\begin{tabular}{|c|c|}
\hline Covariate & Value \\
\hline \multicolumn{2}{|l|}{ Gender } \\
\hline Male & $44.5 \%$ \\
\hline Female & $55.5 \%$ \\
\hline \multicolumn{2}{|l|}{ Highest maternal education level } \\
\hline 0 - grade school & $24.5 \%$ \\
\hline 1 - high school or equivalency ${ }^{a}$ & $20 \%$ \\
\hline 2 - vocational/technical school & $9 \%$ \\
\hline 3 - some college, no degree & $35.5 \%$ \\
\hline 4 - associate degree & $11 \%$ \\
\hline BMI & $19.66(5.31)$ \\
\hline Age & $9.83(3.03)$ \\
\hline \multicolumn{2}{|l|}{ Puberty stage } \\
\hline 1 & $45 \%$ \\
\hline 2 & $13 \%$ \\
\hline 3 & $24.5 \%$ \\
\hline 4 & $13 \%$ \\
\hline 5 & $4.5 \%$ \\
\hline $\mathrm{TL}$ & $1.7(0.46)$ \\
\hline \multicolumn{2}{|l|}{ DHEA reactivity } \\
\hline Baseline & $3.68(1.28)$ \\
\hline Peak & $3.77(1.23)$ \\
\hline Recovery & $3.9(1.27)$ \\
\hline \multicolumn{2}{|l|}{ DHEA diurnal } \\
\hline Waking & $4.1(1.29)$ \\
\hline Morning & $3.67(1.47)$ \\
\hline Afternoon & $3.8(1.49)$ \\
\hline Evening & 3.55 (1.39) \\
\hline
\end{tabular}

\section{Results}

Raw correlations between predictor and outcome scores are shown in Table 2. TL did not correlate with any of the control items (age, sex, puberty status, BMI, maternal education), nor with any of the DHEA terms (basal day waking level, TSST-C-reactivity, TSST-C-peak, and TSSTC-recovery) except for DHEA diurnal slope $(r=0.257$, $P<0.05)$. Next, DHEA scores were modeled independently as predictors of TL (Table 3). DHEA basal awakening level, reactivity, recovery and peak were not associated with TL, consistent with raw correlations.

DHEA diurnal slope significantly predicted TL $(B=0.257, P<0.05)$. Even after statistically controlling for sex, BMI, age, parental age at conception, and maternal education, a significant effect remained $(B=0.532, P<0.05)$. Parallel models with puberty as the developmental control term instead of age yielded similar results $(B=0.516, P<0.05)$.

Next, interaction terms between puberty and DHEA diurnal slope, and age and DHEA diurnal slope, were created to partial out whether associations with DHEA persisted beyond maturational factors. In the model with DHEA diurnal slope, puberty, and DHEA*puberty, TL was predicted by DHEA $(B=0.473, P=0.084)$ at a trend level. In the model using age and age*DHEA, TL was predicted by DHEA diurnal slope $(B=0.541, P<0.05)$.

Finally, to test whether the effect of DHEA diurnal slope on TL persisted beyond the effects of stress-related DHEA changes, the relationship between DHEA diurnal slope and TL was independently tested when controlling for TSST-C-reactivity slope, TSST-C-recovery slope, and TSST-C-peak DHEA. Across all three models, DHEA diurnal slope persisted as a predictor of TL.

Table 2 Correlation coefficients of terms informing the DHEA-TL models.

\begin{tabular}{|c|c|}
\hline & $\begin{array}{c}\text { DHEA } \\
\text { waking } \\
\text { basal }\end{array}$ \\
\hline DHEA waking basal & 1 \\
\hline DHEA slope basal & 0.097 \\
\hline DHEA TSST-reactivity & 0.059 \\
\hline DHEA TSST-C-recovery & 0.202 \\
\hline DHEA TSST-C-peak & $0.583 * * *$ \\
\hline Mom education & 0.073 \\
\hline Puberty & 0.1 \\
\hline Age & -0.02 \\
\hline BMI & 0.087 \\
\hline TL & -0.038 \\
\hline
\end{tabular}

\begin{tabular}{|c|c|}
\hline $\begin{array}{l}\text { DHEA } \\
\text { slope } \\
\text { basal }\end{array}$ & $\begin{array}{c}\text { DHEA } \\
\text { TSST-C- } \\
\text { reactivity }\end{array}$ \\
\hline 1 & \\
\hline 0.052 & 1 \\
\hline 0.011 & $0.416 * * *$ \\
\hline 0.12 & 0.1 \\
\hline 0.102 & -0.049 \\
\hline 0.08 & -0.172 \\
\hline 0.095 & -0.104 \\
\hline 0.035 & 0.008 \\
\hline 0.257 * & -0.127 \\
\hline
\end{tabular}

\begin{tabular}{|c|c|c|}
\hline $\begin{array}{c}\text { DHEA } \\
\text { TSST-C- } \\
\text { recovery }\end{array}$ & $\begin{array}{c}\text { DHEA } \\
\text { TSST-C- } \\
\text { peak }\end{array}$ & $\begin{array}{l}\text { Maternal } \\
\text { education }\end{array}$ \\
\hline 1 & & \\
\hline $0.223 *$ & 1 & \\
\hline 0.202 & -0.018 & 1 \\
\hline 0.158 & 0.08 & 0.093 \\
\hline 0.105 & 0.015 & 0.027 \\
\hline 0.161 & 0.066 & 0.186 \\
\hline-0.145 & -0.057 & -0.014 \\
\hline
\end{tabular}

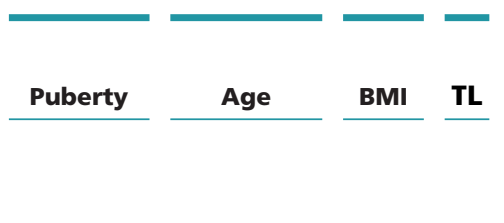

$* P<0.05, * * * P<0.001$.

http://www.endocrineconnections.org DOI: 10.1530/EC-16-0007
This work is licensed under a Creative Commons Attribution-NonCommercial-NoDerivs 4.0 International License. 
Table 3 Beta weights and standard errors of DHEA and covariates predicting buccal TL length.

\begin{tabular}{|c|c|c|c|c|c|}
\hline & DHEA diurnal waking & DHEA diurnal slope & DHEA TSST-C-reactivity & DHEA TSST-C-recovery & DHEA TSST-C-peak \\
\hline DHEA term & $-0.002(0.004)$ & $0.516(0.255) *$ & $-0.013(0.009)$ & $-0.018(0.012)$ & $-0.002(0.004)$ \\
\hline puberty & $-0.001(0.004)$ & $-0.002(0.004)$ & $-0.004(0.003)$ & $-0.003(0.003)$ & $-0.003(0.003)$ \\
\hline BMI & $0.001(0.001)$ & $0.001(0.001)$ & $0.001(0.001)$ & $0.001(0.001)$ & $0.001(0.001)$ \\
\hline Sex & $0.002(0.008)$ & $0.004(0.008)$ & $-0.002(0.007)$ & $-0.002(0.007)$ & $-0.002(0.007)$ \\
\hline Maternal education & $-0.001(0.003)$ & $-0.002(0.003)$ & $-0.001(0.003)$ & $0.000(0.003)$ & $-0.001(0.003)$ \\
\hline
\end{tabular}

$* P<0.05$.

\section{Discussion}

Flatter DHEA diurnal slope was associated with longer $\mathrm{TL}$, and this link persisted even after controlling for age, BMI, sex, maternal education, puberty, and parental age at conception. Furthermore, the relationship between diurnal slope and TL persisted even after accounting for variation in DHEA stress reactivity levels. These are the first results, to our knowledge, to demonstrate an association between these two developmentally sensitive and stressresponsive biomarkers linked to the aging process. That this association was observed within youth adds further to the novelty of this link.

Longer TL was associated with a flatter diurnal slope of DHEA, which is indicative of a stable elevation of DHEA concentrations over the course of a day. Findings were specific to the diurnal DHEA slope and not other DHEA reactivity measures or basal values. That this positive relationship occurs in youth, and is manifested along the diurnal rhythm, rather than acute reactivity, highlights the potential for possible shared mechanistic relationship(s) between DHEA and TL, as described below. The persistence of this finding when examining the interaction with pubertal status and age suggests that DHEA and TL are related, beyond specific developmental contexts, permitting the elaboration of tentative molecular models based on known properties of DHEA and TL regulation. Three potential pathways are described below.

\section{Pathway 1: DHEA as a protective factor against oxidative stress}

DHEA and TL may be linked through oxidative stress. Oxidative stress refers to the damaging effects of reactive oxygen species on a range of cellular components. These damaging effects are mitigated by antioxidants, which serve to delay or prevent oxidation. Oxidative stress accelerates telomere shortening, which increases cellular aging, while DHEA exerts antioxidant properties (22), which may attenuate telomere shortening. Higher DHEA levels throughout the day may buffer or protect the individual from the negative effect of oxidative stress on $\mathrm{TL}$, and other subsequent biological systems known to deteriorate with aging.

\section{Pathway 2: the antiglucocorticoid hypothesis}

In vitro cortisol exposure is associated with decreased telomerase (23), the enzyme responsible for repairing telomere loss. Furthermore, flatter diurnal cortisol levels and elevated cortisol stress reactivity in adults have been associated with shorter TL (24). Evidence suggests that DHEA operates as an antiglucocorticoid agent ameliorating the molecular costs associated with mounting a cortisol response (25). If DHEA attenuates cortisol then increased DHEA across the day (e.g. flatter diurnal slope) would be expected to result in longer TL. Elevated evening levels of cortisol have been associated with poorer health outcomes as well as early life adversity (26). Elevated DHEA levels, particularly late in the day, may be particularly effective at mitigating the negative effects of heightened evening cortisol on TL, thereby providing some protection against cellular aging and subsequent health outcomes.

\section{Pathway 3: ERß activity}

A third molecular pathway that may explain the observed relationship between DHEA and TL is through interactions with other hormone receptors, specifically estrogen receptors. DHEA binds to the estrogen receptor beta (ER $\beta)$ with high affinity (27). When ER $\beta$ is bound to DHEA, or estrogen, it is translocated to the nucleus where it binds to specific estrogen response elements (ERE) in DNA, thereby influencing gene expression $(28,33)$. Located within the regulatory region of telomerase is an ERE that, when stimulated, results in increased telomerase and subsequent attenuation of telomere shortening $(29,30)$. It is plausible that elevated levels of DHEA could bind to 
the $E R E$ in telomerase and increase the downstream level of this enzyme, thus exerting putative buffering effects on the health outcomes associated with shorter telomeres.

DHEA may also be protective by influencing the ER $\beta$ pathway indirectly as this hormone can also be enzymatically converted to testosterone, and subsequently aromatized to estrogen. Estrogen, like DHEA, would be predicted to exert its effects on TL through the reduction of oxidative stress (i.e. pathway 1) or through binding to the estrogen response elements via interactions with ER $\beta$. Consistent with this idea, estrogen deficiency has been linked to shorter telomeres, and higher maternal estrogen levels during pregnancy have been associated with longer infant TL $(30,31)$. Testosterone, an additional downstream product of DHEA, has also been previously associated with TL $(17,32)$. In our analyses, diurnal DHEA predicted TL even when controlling for testosterone, suggesting greater plausibility for alternative pathways. These molecular pathways, while plausible and supported by empirical research, are neither mutually exclusive, nor exhaustive, and other mechanisms may emerge. Rather, the pathways above are presented to emphasize the interpretation that stable, elevated level of DHEA across the day exerts a protective or buffering effect on TL is consistent with known biological pathways and protective influences on TL.

In the current study, the association between DHEA and TL was specific to the diurnal rhythm, and did not manifest across the TSST-C. Prior research has found that stress-reactive vs stable DHEA elevations operate very differently within youth, with basal levels more protective than stress-reactive levels (2). Our findings reinforce this idea, as the association between DHEA and TL in our sample is related to a relatively stable elevation in DHEA level, and not the ability of DHEA to rise in response to a stress event. This is further reinforced by the fact that DHEA diurnal rhythm predicted longer TL even when including DHEA TSST-C-reactivity, -recovery, and -peak values as predictors. The specificity of the association with the diurnal levels and TL increases the relative likelihood of the proposed (and possibly interacting) molecular pathways 1 and 3, which are not dependent on DHEA as a stress-reactive biomarker. Importantly, we used innovative statistical modeling that allow for a nuanced unpacking of DHEA level through the utilization of multiple Bayesian terms to account for changing DHEA concentrations both diurnally and across the stress event, which allows for a specifying individual components of a growth curve. This modeling strategy resolves DHEA into distinct components that can be individually used to predict the outcome of interest, thereby permitting greater resolution of the change in relationships across time.

\section{Limitations}

Limitations to this study exist. First, this study included only African-American youth, thus limiting generalizability to other racial groups. Future examinations would benefit from the inclusion of multiple races in analysis. DHEA-S, the sulfated ester form of DHEA, was not measured. Although plasma levels of DHEA-S are much higher than DHEA, DHEA-S is less biologically active, shows large sex differences, and has a more limited diurnal variation. Secondly, the sample size was not sufficient to see effects of DHEA slope above trend level in the most complex nonparsimonious models.

\section{Conclusions}

The flattened DHEA diurnal slope is associated with longer telomeres in youth. As this is the first study to link DHEA and TL, three putative molecular pathways underlying their association are presented, including oxidative stress, antiglucocorticoid effects, and action via ER $\beta$ receptor and estrogen. Individuals with flatter diurnal rhythms are speculated to have greater stability of DHEA levels across the day, likely enhancing DHEA's protective cellular buffering and decreasing the impact of the cumulative effect of day-to-day challenges. This line of research extends the discussion of cellular and physiological aging to include adolescence as a novel developmental period, where factors linked to the aging process may have substantial, previously unexplored relevance given the reorganization of multiple biological systems resulting in elevated sensitivity to environmental factors. DHEA's role as a potential protective factor mitigating TL decline may inform future basic and clinical research focused on delineating the relationship between stress, aging, and health. As both DHEA and TL have been associated with a range of health outcomes that are accentuated with stress exposure, such as cognitive decline, obesity, and cardiovascular disease $(1,15)$, understanding how these two physiological processes interact has important implications for both normal development and healthy aging while informing future targeted interventions.

Declaration of interest

A R D reports no biomedical financial interests or potential conflicts of interest. S S D reports no biomedical financial interests or potential 
conflicts of interest. K P T reports no biomedical financial interests or potential conflicts of interest. $\mathrm{V} J \mathrm{M}$ reports no biomedical financial interests or potential conflicts of interest. K C E reports no biomedical financial interests or potential conflicts of interest. E A S has a patent in preparation in affiliation with Oasis Diagnostics. E A S has no further financial disclosure.

\section{Funding}

The authors would like to acknowledge the Tulane Oliver Fund (Drury), the Eunice Kennedy Shriver National Institute of Child Health \& Human Development (Award Number K12HD043451, Drury), and the National Institute of Environmental and Health Sciences (R01ES020447, Theall). The content is solely the responsibility of the authors and does not necessarily represent the official views of the Eunice Kennedy Shriver National Institute of Child Health \& Human Development or the National Institutes of Health.

\section{References}

1 Havelock JC, Auchus RJ \& Rainey WE. The rise in adrenal androgen biosynthesis: adrenarche. Seminars in Reproductive Medicine 200422 337-347.

2 Shirtcliff EA, Zahn-Waxler C, Klimes-Dougan B \& Slattery M. Salivary dehydroepiandrosterone responsiveness to social challenge in adolescents with internalizing problems. Journal of Child Psychology and Psychiatry, and Allied Disciplines 200748 580-591. (doi:10.1111/ j.1469-7610.2006.01723.x)

3 Maninger N, Wolkowitz OM, Reus VI, Epel ES \& Mellon SH. Neurobiological and neuropsychiatric effects of dehydroepiandrosterone (DHEA) and DHEA sulfate (DHEAS). Frontiers in Neuroendocrinology 200930 65-91. (doi:10.1016/j.yfrne.2008.11.002)

4 Ye J, Renault VM, Jamet K \& Gilson E. Transcriptional outcome of telomere signalling. Nature Reviews Genetics 201415 491-503. (doi:10.1038/nrg3743)

5 Kroboth PD, Salek FS, Pittenger AL, Faban TJ \& Frye RF. DHEA and DHEA-S: a review. Journal of Clinical Pharmacology 199939 327-348. (doi:10.1177/00912709922007903)

6 Chen JR, Tseng GF, Wang YJ \& Wang TJ. Exogenous dehydroisoandrosterone sulfate reverses the dendritic changes of the central neurons in aging male rats. Experimental Gerontology 201457 191-202. (doi:10.1016/j.exger.2014.06.010)

7 Ohlsson C, Vandenput L \& Tivesten Å. DHEA and mortality: what is the nature of the association? Journal of Steroid Biochemistry and Molecular Biology 2015145 248-253. (doi:10.1016/ j.jsbmb.2014.03.006)

8 Baulieu EE, Thomas G, Legrain S, Lahlou N, Roger M, Debuire B \& Latour F. Dehydroepiandrosterone (DHEA), DHEA sulfate, and aging: contribution of the DHEAge Study to a sociobiomedical issue. PNAS 200097 4279-4284. (doi:10.1073/pnas.97.8.4279)

9 Scheffers CS, Armstrong S, Cantineau AE, Farquhar C \& Jordan V. Dehydroepiandrosterone for women in the peri- or postmenopausal phase. Cochrane Database of Systematic Reviews 2015 1 CD011066.

10 Porsova-Dutoit I, Sulcova J \& Starka L. Do DHEA/DHEAS play a protective role in coronary heart disease? Physiological Research 2000 49 S43-S56.

11 Frenck RW, Blackburn EH \& Shannon KM. The rate of telomere sequence loss in human leukocytes varies with age. PNAS 199895 5607-5610.

12 Chen W, Kimura M, Kim S, Cao X, Srinivasan SR, Berenson GS \& Aviv A. Longitudinal versus cross-sectional evaluations of leukocyte telomere length dynamics: age-dependent telomere shortening is the rule. Journals of Gerontology Series A: Biological Sciences and Medical Sciences 201166 312-319. (doi:10.1093/gerona/glq223)
13 Epel ES, Lin J, Wilhelm FH, Wolkowitz OM, Cawthon R, Adler NE \& Blackburn EH. Cell aging in relation to stress arousal and cardiovascular disease risk factors.

Psychoneuroendocrinology 200631 277-287. (doi:10.1016/ j.psyneuen.2005.08.011)

14 Hamel FG. Telomeres and type 2 diabetes. Translational Research 2010 155 164-165. (doi:10.1016/j.trsl.2009.12.004)

15 Shalev I, Entringer S, Wadhwa PD, Wolkowitz OM, Puterman E, Lin J \& Epel ES. Stress and telomere biology: a lifespan perspective. Psychoneuroendocrinology 201338 1835-1842. (doi:10.1016/ j.psyneuen.2013.03.010)

16 Heidinger BJ, Blount JD, Boner W, Griffiths K, Metcalfe NB \& Monaghan P. Telomere length in early life predicts lifespan. PNAS 2012109 1743-1748. (doi:10.1073/pnas.1113306109)

17 Drury SS, Shirtcliff EA, Shachet A, Phan J, Mabile E, Brett ZH $\&$ Theall KP. Growing up or growing old? Cellular aging linked with testosterone reactivity to stress in youth. American Journal of the Medical Sciences 2014348 92-100. (doi:10.1097/ MAJ.0000000000000299)

18 Buske-Kirschbaum A, Jobst S, Wustmans A, Kirschbaum C, Rauh W \& Hellhammer D. Attenuated free cortisol response to psychosocial stress in children with atopic dermatitis. Psychosomatic Medicine 199759 419-426. (doi:10.1097/00006842199707000-00012)

19 Petersen A, Crockett L, Richards M \& Boxer A. A self-report measure of pubertal status: reliability, validity, and initial norms. Journal of Youth and Adolescence 198817 117-133. (doi:10.1007/ BF01537962)

20 Shirtcliff EA, Dahl RE \& Pollak SD. Pubertal development: correspondence between hormonal and physical development. Child Development 200980 327-337. (doi:10.1111/j.14678624.2009.01263.x)

21 De Meyer T, Rietzschel ER, De Buyzere ML, De Bacquer D, Van Criekinge W, De Backer GG \& Bekaert S. Paternal age at birth is an important determinant of offspring telomere length. Human Molecular Genetics 200716 3097-3102. (doi:10.1093/ hmg/ddm271)

22 Bastianetto S, Ramassamy C, Poirier J \& Quirion R. Dehydroepiandrosterone (DHEA) protects hippocampal cells from oxidative stress-induced damage. Molecular Brain Research 199966 35-41. (doi:10.1016/S0169-328X(99)00002-9)

23 Choi J, Fauce SR \& Effros RB. Reduced telomerase activity in human T lymphocytes exposed to cortisol. Brain, Behavior, and Immunity 2008 22 600-605. (doi:10.1016/j.bbi.2007.12.004)

24 Tomiyama AJ, O’Donovan A, Lin J, Puterman E, Lazaro A, Chan J \& Blackburn E. Does cellular aging relate to patterns of allostasis? An examination of basal and stress reactive HPA axis activity and telomere length. Physiology \& Behavior 2012106 40-45. (doi:10.1016/j.physbeh.2011.11.016)

25 Kalimi M, Shafagoj Y, Loria R, Padgett D \& Regelson W. Antiglucocorticoid effects of dehydroepiandrosterone (DHEA). Molecular and Cellular Biochemistry 1994131 99-104. (doi:10.1007/BF00925945)

26 Shirtcliff EA, Allison AL, Armstrong JM, Slattery MJ, Kalin NH \& Essex MJ. Longitudinal stability and developmental properties of salivary cortisol levels and circadian rhythms from childhood to adolescence. Developmental Psychobiology 201254 493-502. (doi:10.1002/dev.v54.5)

27 Webb SJ, Geoghegan TE, Prough RA \& Miller KK. The biological actions of dehydroepiandrosterone involves multiple receptors. Drug Metabolism Reviews 200638 89-116. (doi:10.1080/03602530600569877)

28 Bruder JM, Sobek L \& Oettel M. Dehydroepiandrosterone stimulates the estrogen response element. Journal of Steroid Biochemistry and Molecular Biology 199762 461-466. (doi:10.1016/S09600760(97)00056-3) 
29 Kyo S, Takakura M, Kanaya T, Zhuo W, Fujimoto K, Nishio Y \& Inoue M. Estrogen activates telomerase. Cancer Research 199959 5917-5921.

30 Entringer S, Epel ES, Lin J, Blackburn EH, Buss C, Simhan HN \& Wadhwa PD. Maternal estriol (E3) concentrations in early gestation predict infant telomere length. Journal of Clinical Endocrinology \& Metabolism 2014100 267-273.

31 Bayne S, Jones ME, Li H, Pinto AR, Simpson ER \& Liu JP. Estrogen deficiency leads to telomerase inhibition, telomere shortening and reduced cell proliferation in the adrenal gland of mice. Cell Research 200818 1141-1150. (doi:10.1038/cr.2008.291)

32 Desai N, Sabanegh E, Kim T \& Agarwal A. Free radical theory of aging: implications in male infertility. Urology 201075 14-19. (doi:10.1016/j.urology.2009.05.025)

33 Gruber CJ, Gruber DM, Gruber IML, Wieser F \& Huber JC. Anatomy of the estrogen response element. Trends in Endocrinology \& Metabolism 200415 73-78.

Received in final form 11 March 2016

Accepted 4 April 2016
This work is licensed under a Creative Commons Attribution-NonCommercial-NoDerivs 4.0 International License. 